\title{
Modeling of Random Channel Parameter Variations in MOS Transistors
}

\author{
Mao-Feng Lan and Randall Geiger \\ Department of Electrical and Computer Engineering \\ Iowa State University, Ames, IA 50011, U.S.A. \\ Email: rlgeiger@iastate.edu
}

\begin{abstract}
Widely used approaches to modeling random effects and extracting random parameters in matching-critical circuits are based upon models derived under the widely accepted premise that distributed parameter devices can be modeled with lumped parameter models. In this paper, a new stochastic approach based upon a distributed parameter model is presented that offers improvement in predicting the effects of random parameter variations on device matching.
\end{abstract}

\section{INTRODUCTION}

It is well recognized that the performance of many linear and mixed-signal integrated circuits such as current mirrors and differential amplifiers is limited by how well the I/V characteristics of transistors can be matched. The impact of mismatch is of increasing concern as attempts are made to reduce supply voltages, decrease feature sizes, reduce power and increase the frequency of operation of the analog circuitry on a chip. The matching performance of basic circuit elements is attributable to both systematic and random variations in geometric parameters, process parameters and device parameters [1][2]. Conventional wisdom suggests that random variations are easy to model and tradeoffs can be made between area and performance to compensate for random parameter variations [1][2]. This observation is referred as law of area and can be expressed as

$$
\sigma^{2}(\gamma)=\frac{A_{\gamma}^{2}}{\text { Area }}
$$

where $\gamma$ can be model or process parameters and $\mathrm{A}_{\gamma}$ is an area proportionality constant [2]. Variations can be discussed for one single device, e.g. $\gamma$, or a differential pair of devices, e.g. $\Delta \gamma$, and thus it is important to distinguish between the two clearly. $\mathrm{A}_{\gamma}$ and $\mathrm{A}_{\Delta \gamma}$ will be used to represent the area proportionality constants on variations for one single device and a differential pair respectively and their relationship can be expressed as

$$
A_{\Delta \gamma}=\sqrt{2} A_{\gamma}
$$

If $\sigma(\gamma)$ and $\sigma(\Delta \gamma)$ are plotted as a function of $1 / \sqrt{\text { Area }}, \mathrm{A}_{\gamma}$ and $\mathrm{A}_{\Delta \gamma}$ will be the slope of the curves respectively [2]. $\sigma(\gamma)$ and $\sigma(\Delta \gamma)$ are basically extracted from the measurement of $\mathrm{I} / \mathrm{V}$ characteristics and the extraction formula used in the triode region can be expressed as

$$
I_{D}=\beta \frac{\left(V_{G S}-V_{T}-\frac{V_{D S}}{2}\right) V_{D S}}{1+\boldsymbol{\theta}\left(V_{G S}-V_{T}\right)}
$$

where $\beta=\mathrm{C}_{\mathrm{OX}} \mu \mathrm{W} / \mathrm{L}$ is the current factor and $\theta$ is responsible for the mobility degradation effect [2]. $\gamma$ in (1) can be the threshold voltage $\left(\mathrm{V}_{\mathrm{T} 0}\right)$, the mobility $(\mu)$, and the gate oxide capacitance density (Cox), etc. Because the extracted $\mathrm{A}_{\gamma}$ will be used to represent variation characteristics of the process, it should be process dependent only and independent of the shapes of devices. However, a current research [3] showed there was a significant difference in $A_{V T}$ 's measured with hexagonal and rectangular transistors. This observation flags a warning sign on the process of extraction because the extracted value does not truly represent the inherent variation characteristics of the process and this discrepancy is mainly due to the extraction process based upon lumped models. Thus we propose another approaches to model the random variation from the viewpoint of the distributed domain and this approach can explain some unexpected observations on random variations.

\section{CONSISTENT MODEL FOR RANDOM PARAMETER VARIATIONS}

In the traditional approach, the random model parameters are extracted directly from $\mathrm{I} / \mathrm{V}$ device measurements based upon the lumped model of (3). With the assumption that the integral model characterizes the equivalent model parameters, a model for the random parameter variance that is characterized by a single model parameter, such as $\mathrm{A}_{\mathrm{VT}}$, and depends only upon the reciprocal of the channel area was derived. This model was fit to the measured data to obtain the model parameter $A_{\mathrm{VT}}$. This approach which showed a reciprocal area dependence of the variance of the threshold voltage was reassuring since the randomness in processing such as the randomness in the position of ions implanted in a channel region or the randomness of impurities during crystal growth also show a reciprocal area dependence on the variance. Unfortunately, this approach resulted in an inconsistent model which diverged when the model was applied to a segmented transistor.

In an attempt to obtain a more accurate model of the effects of random variations in the threshold voltage on circuit performance, we will retain the assumption that the variance of the threshold voltage exhibits a reciprocal area dependence but revisit how this process parameter affects the device performance by returning to the gradual channel approximation which was used to obtain the IV characteristics of the device. For convenience, the device is assumed to be rectangular. A cross section of a device operating in saturation is shown in Fig. 1a. For notational convenience, it will be assured that the reference node is the source and thus $\mathrm{V}_{\mathrm{GS}}=\mathrm{V}_{\mathrm{G}}$ and $\mathrm{V}(\mathrm{ys})=\mathrm{V}(\mathrm{y})$.

In the gradual channel approximation, the channel voltage at position $\mathrm{y}$ in the channel when operating in saturation, $\mathrm{V}(\mathrm{y})$, can be expressed as

$V(y)=\left(V_{G S}-V_{T}\right)\left(1-\sqrt{1-\frac{y}{L}}\right)$

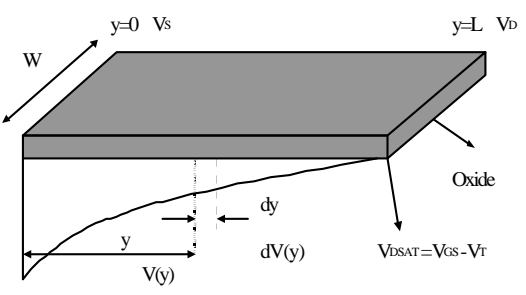

(a)

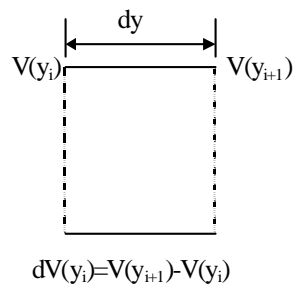

(b)
Fig. 1 Cross section of a device

The effects of random variations in $\mathrm{V}_{\mathrm{T}}$ on the drain current will now be investigated. If the threshold voltage is non-stochastic, the process parameter $\mathrm{V}_{\mathrm{T}}$ is also a model parameter. If the process threshold voltage becomes stochastic, the effects of $\mathrm{V}_{\mathrm{T}}$ on the model also become stochastic. If the same variable is used for both the process threshold voltage and the model threshold voltage, the variables are inherently assumed to be identical and thus have identical probability density functions. If this assumption is made and if it is assumed that the variance of $V_{T}$ has a 
reciprocal area dependence, it was shown that the resultant device model was inconsistent [4]. Thus, the question naturally arises - Is the process threshold voltage identical to the model threshold voltage? To address this question, in this section the variable $\mathrm{V}_{\mathrm{T}}$ will refer to the process threshold voltage and $\mathrm{V}_{\mathrm{TM}}$ will refer to the model threshold voltage. The process threshold voltage for a $\mathrm{n}$-channel transistor will be defined by the relationship

$Q_{A}=C_{O X}\left(V_{G C}-V_{T}\right) A$

where $\mathrm{Q}_{\mathrm{A}}$ is the channel charge in a channel region of area $\mathrm{A}$ and $\mathrm{V}_{\mathrm{GC}}$ is the gate-channel voltage which is assured constant throughout the channel region. It will be assured that the random variable $\mathrm{V}_{\mathrm{T}}$ has a mean $\mathrm{V}_{\mathrm{TN}}$ and a variance given by

$\sigma_{V T}^{2}=\frac{\tilde{A}_{V T}^{2}}{A}$

where $\widetilde{\mathrm{A}}_{V T}$ is a constant characteristic of the process.
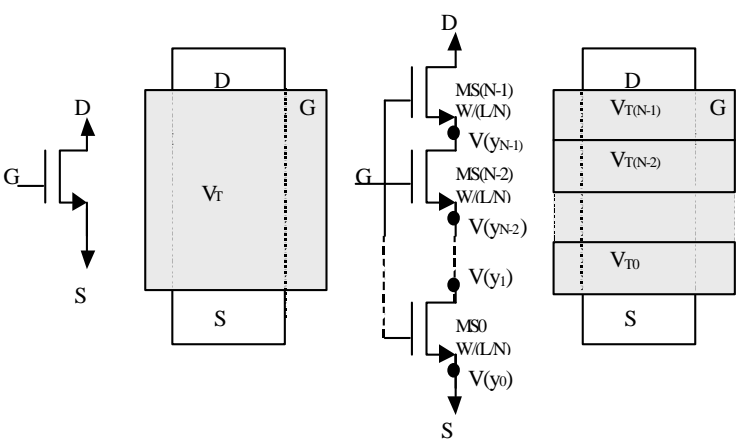

(a)

(b)

Fig 2. Two equivalent representations of a transistor a) Single segment, b) N-Segment

If we now consider a segment in the channel of a transistor at location $\mathrm{y}$ and of length dy as depicted in Fig. 2, it follows from the notation of Fig. 1 that the charge density can be expressed as

$Q(y)=C_{O X}\left(V_{G S}-V_{T}-V(y)\right)$

If the port variables are assured fixed, it follows from (7) that

$\sigma^{2}(Q(y))=C_{O X}^{2} \sigma^{2}\left(V_{T}\right)$

and from (6) that

$\sigma^{2}(Q(y))=C_{O X}^{2} \frac{\tilde{A}_{V T}^{2}}{A}$

If $\mathrm{V}_{\mathrm{T}}$ in (7) is assured to be stochastic, it can be expressed as $V_{T}=V_{T N}+V_{T R}$

where $\mathrm{V}_{\mathrm{TN}}$ is the nominal value of the threshold voltage and $\mathrm{V}_{\mathrm{TR}}$ is the random component of the threshold voltage. It thus can be expressed as

$I_{D} d y=\mu C_{O X} W\left(V_{G S}-V_{T N}-V_{T R}-V(y)\right) d V(y)$

If this is now integrated from the drain to source of the segmented transistor, we obtain

$$
\begin{aligned}
& I_{D}=\frac{\mu C_{O X} W}{d y}\left(V_{G S}-V_{T N}-\frac{V(y+d y)-V(y)}{2}\right)(V(y+d y)-V(y))_{(12)} \\
& +\frac{\mu C_{O X} W}{d y} \int_{V(y)}^{V(y+d y)} V_{T R} d V(y)
\end{aligned}
$$

If we now assume that

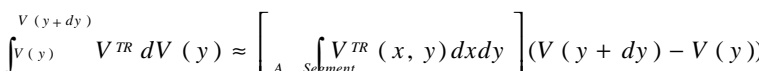

then $I_{D}$ can be expressed as
$I_{D}=\frac{\mu C_{O X} W}{d y}\left(V_{G S}-V_{T M}-\frac{V(y+d y)-V(y)}{2}\right)(V(y+d y)-V(y))$

where the model threshold voltage, $\mathrm{V}_{\mathrm{TM}}$, satisfies the relation

$V_{T M}=V_{T N}+\left[\int_{A_{-} \text {Segment }} V_{T R}(x, y) d x d y\right]$

This is the standard square law model for the segmented transistor. It follows from (60) that

$\sigma^{2}\left(I_{D}\right)=\left(\frac{\mu C_{O X} W}{d y}\right)^{2}(V(y+d y)-V(y))^{2} \sigma^{2}\left(V_{T M}\right)$

but from (8) that it can be shown

$\sigma^{2}\left(I_{D}\right)=\left(\frac{\mu C_{O X} W}{d y}\right)^{2}(d V(y))^{2} \sigma^{2}\left(V_{T}\right)$

From (16) and (17), it thus follows that

$\sigma^{2}\left(V_{T M}\right)=\sigma^{2}\left(V_{T}\right)$

It can be shown that the expected values of $\mathrm{V}_{\mathrm{T}}$ and $\mathrm{V}_{\mathrm{TM}}$ also agree for the segmented transistor. The model of the threshold voltage of (17) was used for the simulations, $\mathrm{V}_{\mathrm{GS}}=2.5 \mathrm{~V}, \mathrm{~V}_{\mathrm{DS}}=1.7 \mathrm{~V}$, and $\mathrm{V}_{\mathrm{TN}}=0.8 \mathrm{~V}$, that were summarized in Fig. 3 and this resulted in an inconsistent model. It should be noted that the relationship of (17) is a direct consequence of the relationship of (8) which was obtained since the port variables in the segment model were assumed fixed.

It should be observed, however, that in an actual transistor, the charge density is strongly dependent upon the position in he channel. Equation (8) obtained under the assumption that the port voltages are not stochastic, indicates that the variance in the channel charge density is independent of position since the statistics of the process threshold voltages are independent of position. However, it can be observed from (4) and (7) that

$Q(y)=C_{o x}\left(V_{G S}-V_{T}\right) \sqrt{1-\frac{\mathrm{y}}{\mathrm{L}}}$

Thus

$\sigma^{2}(Q(y))=C_{o x}^{2}\left(1-\frac{y}{L}\right) \sigma^{2}\left(V_{T}\right)$

or, equivalently from (4)

$\sigma^{2}(Q(y))=C_{O X}^{2}\left(1-\frac{V(y)}{V_{G S}-V_{T}}\right)^{2} \sigma^{2}\left(V_{T}\right)$

Note that (20) differs considerably from (8) obtained under the assumption that the port variables were non-stochastic. It follows from (20) that

$\sigma^{2}\left(I_{D}\right)=\frac{\mu^{2} C_{O X}^{2} W^{2}\left(1-\frac{y}{L}\right)}{(d y)^{2}} \sigma^{2}\left(V_{T}\right)$

Thus from (16), (22) and (6), we obtain

$\sigma^{2}\left(V_{T M}\right)=\left(1-\frac{y}{L}\right) \frac{\tilde{A}_{V T}^{2}}{A}$

or, equivalently

$\sigma^{2}\left(V_{T M}\right)=\left(1-\frac{V(y)}{V_{G S}-V_{T}}\right) \frac{\tilde{A}_{V T}^{2}}{A}$

Equations (23) and (24) are equivalent when the device is operating in the saturation region. If the transistor is operating in the triode region, (6) no longer applies but it can be shown that (20) and correspondingly (24) remain valid.

From (18) and (23), it is apparent that the probability density function of the model parameter, $\mathrm{V}_{\mathrm{TM}}$, differs from that of the process parameter $\mathrm{V}_{\mathrm{T}}$ and this difference is very significant as the saturated part of the channel is approached.

The model of (23) was used to model the stochastic threshold voltage for the segmented transistors in HSpice. The simulation results for the drain current standard deviation of a single transistor are shown in Fig. 4 for the same test condition used in the Fig. 3. 


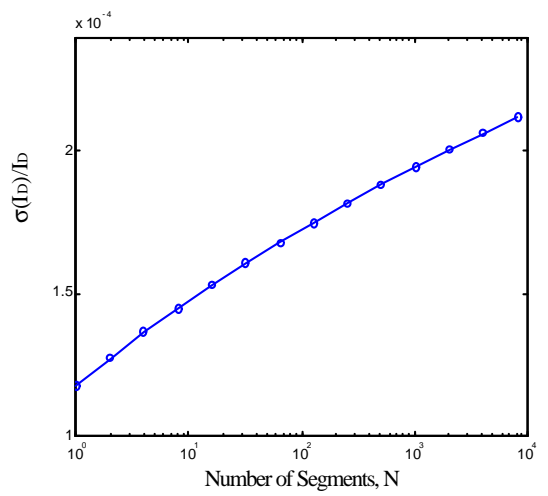

Fig 3. $\sigma\left(\mathrm{I}_{\mathrm{D}}\right) / \mathrm{I}_{\mathrm{D}}$ for a segmented transistor

It can be seen that the results now converge. The issue of what value of variance actually characterizes the device must be addressed since the variance is dependent upon the number of segments used in the simulation. The value is the asymtotic value shown in the figure. If a small number of segments are used, the channel voltage dependence on $\mathrm{V}_{\mathrm{TM}}$ is quantified into a small number of bins and this quantization biases the simulation results.

Also shown in the figure are the simulation results for a transistor operating in the triode region biased at $\mathrm{V}_{\mathrm{GS}}=2.5 \mathrm{~V}, \mathrm{~V}_{\mathrm{DS}}=1 \mathrm{~V}$, and $\mathrm{V}_{\mathrm{TN}}=0.8 \mathrm{~V}$. It can be observed that it converges to the same value as was obtained for the saturated channel device, This indicates that the normalized variance in the drain current is not operation region dependent. If, however, a single-segment model is used, it can be shown analytically that the statistics for $I_{D}$ are strongly operating point dependent and vary considerably between the triode and saturation regions. This can be seen as well in the plot of Fig. 4 in the case where $\mathrm{N}=1$.

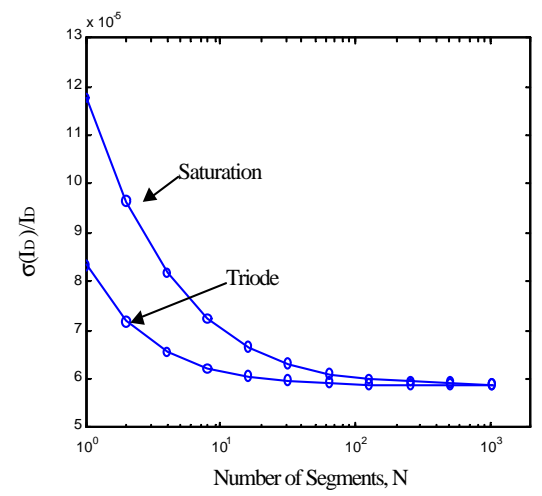

Fig. 4 Current mismatch for a single device operating in saturation and triode regions with the model of (24)

The issue of consistency in the variance model of (20) and (24) also deserves consideration. Simulations of two series connected transistors have been made and the results show convergence to the same values as was obtained for the single equivalent transistor indicating that the model is consistent.

Unfortunately, the time required for a Spice-type simulation needed to obtain convergence is very long because of the large number of segments needed and because of the large number of simulations needed for each value of $\mathrm{N}$. An alternative development that circumvents the need for the large number of Spice simulation has been developed and the current variation can be shown from the notation of Fig. 2 as

$$
\frac{\sigma^{2}\left(I_{D}\right)}{I_{D_{-} \text {Nom }}^{2}}=\left(\frac{1}{N}\right)^{2} \sum_{i=0}^{N-1} \frac{\sigma_{i}^{2}\left(V_{T}\right)\left(1-\frac{y_{i}}{L}\right)}{\left(V_{G}-V\left(y_{i}\right)-V_{T N}-\frac{1}{2} d V\left(y_{i}\right)\right)^{2}}
$$

where $\sigma_{i}\left(V_{T}\right)$ is the $V_{T}$ variation happening within $i$ th region of length dy and width $\mathrm{W}$. However, when $\mathrm{N}$ approaches to the infinity, $\operatorname{dV}\left(\mathrm{y}_{\mathrm{i}}\right)$ will approach zero and thus (25) can be simplified as

$$
\frac{\sigma^{2}\left(I_{D}\right)}{I_{D_{-} N o m}^{2}}=\left(\frac{1}{N}\right)^{2} \sum_{i=0}^{N-1} \frac{\sigma_{i}^{2}\left(V_{T}\right)}{\left(V_{G S}-V_{T N}\right)^{2}}
$$

Although there is an assumption of operating in saturation region, $\mathrm{V}_{\mathrm{s}}=\mathrm{V}_{\mathrm{GS}^{-}} \mathrm{V}_{\mathrm{T}}$, in the beginning, (25) and (26) can also be shown valid within the triode region and $\mathrm{V}(\mathrm{ys})=\mathrm{V}(\mathrm{y})-\mathrm{V}_{\mathrm{S}}$ can be expressed as

$V(y s)=\left(V_{G S}-V_{T}\right)-\sqrt{\left(V_{G S}-V_{T}\right)^{2}-\frac{2 y}{L}\left(\left(V_{G S}-V_{T}\right) V_{D S}-\frac{1}{2} V_{D S}^{2}\right)}(27)$

Next, the validity of model (25) and (26) are going to be verified The device used for the verification is assumed with the size of width $100 \mu \mathrm{m}$ and length $100 \mu \mathrm{m}$ and will be simu lated under two working regions. One is working in the saturation region, $\mathrm{V}_{s}=\mathrm{V}_{\mathrm{GS}^{-}} \mathrm{V}_{\mathrm{T}}$, where $\mathrm{V}=1.7 \mathrm{~V}$, $\mathrm{V}_{\mathrm{G}}=2.5 \mathrm{~V}, \mathrm{~V}_{\mathrm{S}}=0$, and $\mathrm{V}_{\mathrm{T}}=0.8 \mathrm{~V}$, and the other is the triode region where $\mathrm{V}_{\mathrm{D}}=1 \mathrm{~V}, \mathrm{~V}_{\mathrm{G}}=2.5 \mathrm{~V}, \mathrm{~V}_{\mathrm{S}}=0$, and $\mathrm{V}_{\mathrm{T}}=0.8 \mathrm{~V}$. Similarly, the device is partitioned into $\mathrm{N}$ segments and is shown in Fig. 2b. The simulation model is a level 2 device model for $2 \mu \mathrm{m} \mathrm{n}$-well process available through MOSIS except that $\mathrm{V}_{\mathrm{T}}$ is replaced by $0.8 \mathrm{~V}$ and $\tilde{\mathrm{A}}_{V T}$ is assumed as $10 \mathrm{mV} \cdot \mu \mathrm{m}$. The simulation results of (25) and (26) with two working regions are plotted as a function of the number of segments, N, in Fig. 5. The results show there is an excellent agreement between Hspice and Matlab simulation results.

From the Fig. 5, it is apparent that the simulation results with (26) are independent of the number of segments, $\mathrm{N}$, and the results of the approach (25) will converge when $\mathrm{N}$ becomes larger. Also, all curves are essentially coincident for large N. It is also shown in Fig. 5 that the current variations, $\sigma\left(I_{D}\right) / I_{D_{-} N o m}$, within saturation and triode regions have the same asymptotic value with given $\mathrm{V}_{\mathrm{GS}}$ and $\mathrm{V}_{\mathrm{T}}$ although they have different starting points (when $\mathrm{N}=1$ ).

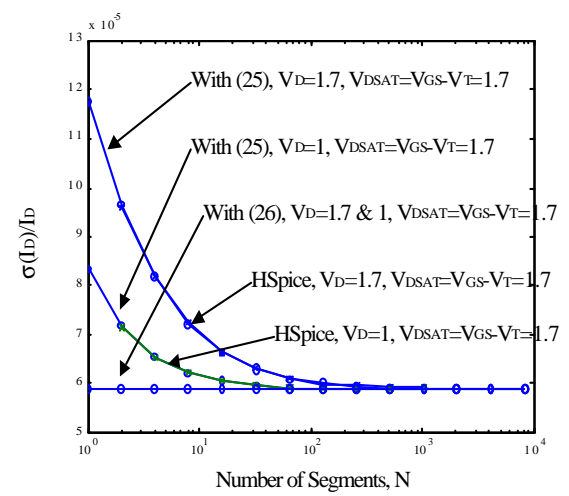

Fig. 5 Current mismatch for a single device using Matlab and Hspice

\section{DISCUSSION}

Equation (25) with $\mathrm{N}=1(\mathrm{y}=0)$ is rewritten as

$$
\frac{\sigma^{2}\left(I_{D}\right)}{I_{D_{-} N o m}^{2}}=\frac{\sigma^{2}\left(V_{T}\right)}{\left(V_{G S}-V_{T N}-\frac{1}{2}\left(V_{D S}\right)\right)^{2}}
$$

Then $\mathrm{V}_{\mathrm{DS}}=\mathrm{V}_{\mathrm{GS}}-\mathrm{V}_{\mathrm{TN}}$ is substituted into (28) and then the following equation will be given

$$
\frac{\sigma^{2}\left(I_{D}\right)}{I_{D_{-} \text {Nom }}^{2}}=\frac{4 \sigma^{2}\left(V_{T}\right)}{\left(V_{G S}-V_{T N}\right)^{2}}
$$

In the traditional approach to modeling random parameters, $\sigma\left(\mathrm{V}_{\mathrm{T}}\right)$ in (28) and (29) can be expressed as

$$
\sigma^{2}\left(V_{T}\right)=\frac{A_{V T}^{2}}{\mathrm{~W} L}
$$


The two equations, (28) and (29), have been widely used to extract model parameters in the triode and saturation regions respectively. According to the simulation results shown in Fig. 5, it shows that the current variation, $\sigma\left(I_{D}\right) / I_{D}$, will be a constant for a given excess voltage within the range, $0 \leq \mathrm{V}_{\mathrm{D}} \leq \mathrm{V}_{\mathrm{GS}}-\mathrm{V}_{\mathrm{T}}$. This implies that the extracted area proportionality constant, $A_{V T}$, will be $V_{D S}$ dependent according to (28) and (29) with a given excess voltage. If current mismatch can be measured and threshold voltage statistics can be inferred from (28) and (29), the measurements will inherently be made on a distributed device and the asymptotic values depicted in Fig. 5. Thus, the parameter $\mathrm{A}_{\mathrm{VT}}$ 's for the equations (28) and (29) that give values that agrees with the measured results is what we need. Then the relationship between $\tilde{\mathrm{A}}_{V T}$ and $\mathrm{A}_{\mathrm{VT}}$ can be derived from (28) and (29) in the triode region as

$\frac{\sigma^{2}\left(I_{D}\right)}{I_{D_{-} \text {Nom }}^{2}}=\frac{\tilde{A}_{V T}^{2}}{W L\left(V_{G S}-V_{T N}\right)^{2}}=\frac{A_{V T}^{2}}{W L\left(V_{G}-V_{S}-V_{T N}-\frac{1}{2}\left(V_{D}-V_{S}\right)\right)^{2}}$

Thus the $\mathrm{A}_{\mathrm{VT}}$ can be expressed in the term of $\tilde{\mathrm{A}}_{V T}$ as

$$
A_{V T}=\left(1-\frac{1}{2} \frac{V_{D S}}{V_{G S}-V_{T N}}\right) \tilde{A}_{V T}
$$

The equation (31) is only valid within the range that the gradual channel approximation can be validly applied. After the device reaches to the deep saturation region, a depleted channel section $\Delta \mathrm{L}$ will be introduced as shown in Fig. 6 and there is almost carrier free within this section. The depletion region, $\Delta \mathrm{L}$, can be derived by the first-order approximation as

$$
\Delta L=\frac{\lambda\left(V_{D S}-V_{D S A T}\right) L}{1+\lambda\left(V_{D S}-V_{D S A T}\right)}
$$

where $\lambda$ is called the channel length modulation parameter and $\mathrm{V}_{\mathrm{DSAT}}=\mathrm{V}_{\mathrm{GS}}-$ $\mathrm{V}_{\mathrm{T}}$ is the pinch-off voltage.

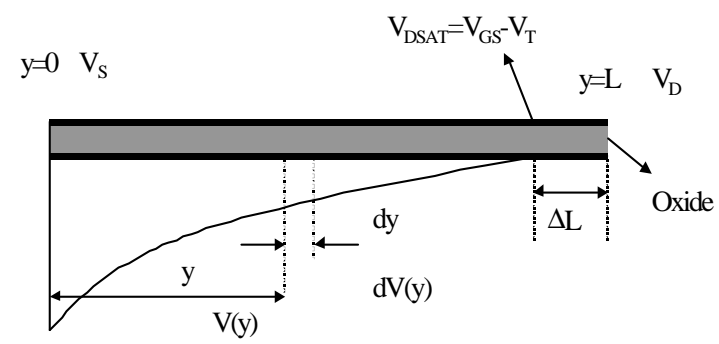

Fig. 6 Cross section of a device in saturation region

Because it is almost carrier free within the depletion region, the depletion region contributes nothing to current variation. Thus current variation in the deep saturation region can be expressed as

$\frac{\sigma^{2}\left(I_{D}\right)}{I_{D_{-} N o m}^{2}}=\frac{\tilde{A}_{V T}^{2}}{W L\left(V_{G S}-V_{T N}\right)^{2}}\left(1-\frac{\Delta L}{L}\right)$

Substituting (33) into (34) and the following expression will be given

$\frac{\sigma^{2}\left(I_{D}\right)}{I_{D_{-} \text {Nom }}^{2}}=\frac{\tilde{A}_{V T}^{2}}{W L\left(V_{G S}-V_{T N}\right)^{2}} \frac{1}{1+\lambda\left(V_{D S}-V_{D S A T}\right)}$

According to (29) and (35), the relationship of $\mathrm{A}_{V T}$ and $\widetilde{\mathrm{A}}_{V T}$ in the deep saturation region can be given as

$$
A_{V T}=\frac{\tilde{A}_{V T}}{2} \sqrt{\frac{1}{1+\lambda\left(V_{D S}-V_{D S A T}\right)}}
$$

$\mathrm{A}_{\mathrm{VT}} / \widetilde{\mathrm{A}}_{V T}$ for (32) and (36) are plotted in Fig. 7 as a function of $\mathrm{V}_{\mathrm{DS}} / \mathrm{V}_{\mathrm{DSAT}}$, and $\widetilde{\mathrm{A}}_{V T}, \mathrm{~V}_{\mathrm{DAST}}$, and $\lambda$ are assumed as $10 \mathrm{mV} \cdot \mu \mathrm{m}, 1.7 \mathrm{~V}$, and $0.05 \mathrm{~V}^{1}$ respectively. In Fig. 7, it shows that there is a linear relationship existing between $A_{V T}$ and $\widetilde{\mathrm{A}}_{V T}$ and after the device is going into the saturation region, the ratio of $\mathrm{A}_{\mathrm{VT}} / \widetilde{\mathrm{A}}_{V T}$ is almost constant. Unfortunately, this observation is usually ignored carelessly during the testing process.

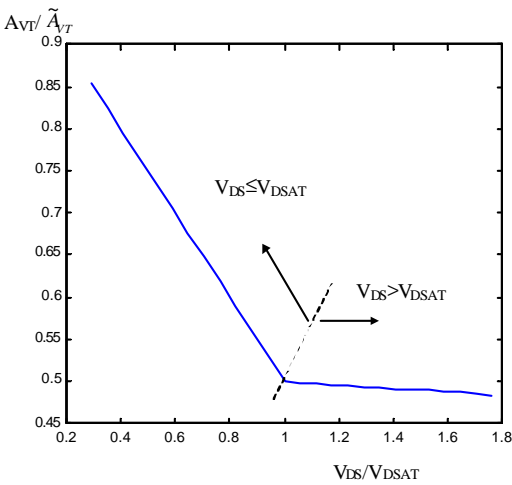

Fig. $7 \mathrm{~A}_{\mathrm{VT}} / \tilde{\mathrm{A}}_{V T}$ in the function of $\mathrm{V}_{\mathrm{DS}} / \mathrm{V}_{\mathrm{DSAT}}$

\section{CONCLUSION}

A new model for characterizing the effects of random variation of model parameters in MOS transistors has been introduced. This model overcomes the inconsistencies inherent in existing approaches of predicting the matching characteristics of devices and matching-critical circuits.

\section{ACKNOWLEDGEMENT}

This work was supported, in part, by National Semiconductor and the R. J. CARVER Trust. Simulation results were obtained, in part, from Avant!'s HSPICE program made available through the company's university program.

\section{REFERENCES}

[1] K. R. Lakshmikumar, R. A. Hadaway, and M. A. Copeland, "Characterization and modeling of mismatch in MOS transistors for precision analog design," IEEE J. Solid-State Circuits, vol. SC-21, pp. 1057-1066, 1986.

[2] M. J. M. Pelgrom, A.C. J. Duinmaijer, and A. P. G. Welbers, "Matching properties of MOS transistors", IEEE J. Solid-State Circuits, vol. SC-24, pp. 1433-1439, 1989.

[3] Anne Van den Bosch, M. S. J. Steyert and W. Sansen, “A High-Density, Matched Hexagonal Transistor Structure in Standard CMOS Technology for High-Speed Applications," IEEE Transactions on Semiconductor Manufacturing, VOL. 13, No. 2, May 2000.

[4] Mao-Feng Lan, and R. L. Geiger, "Impact of Model Errors on Predicting Performance of Matching-Critical Circuits ," in Proc. MWSCAS, Lansing, Michigan, Aug 8-11, 2000. 